\title{
IMPROVED ANALYTICAL BOUNDS FOR SOME RISK QUANTITIES
}

\author{
WERNER HÜRLIMANN \\ Allgemeine Mathematik, Paulstr. 9, Winterthur-Leben
}

\begin{abstract}
Simple analytical lower and upper bounds are obtained for stop-loss premiums and ruin probabilities of compound Poisson risks in case the mean, variance and range of the claim size distribution are known. They are based on stop-loss extremal distributions and improve the bounds derived earlier from dangerous extremal distributions. The special bounds obtained in case the relative variance of the claim size is unknown, but its maximal value is known, are related to other actuarial results.
\end{abstract}

\section{KEYWORDS}

analytical bounds, stop-loss, ruin probability, stochastic orderings, relative variance, atomic distributions

\section{INTRODUCTION}

A main topic of risk theory under incomplete information is the construction of divers bounds for risk quantities using stochastic orderings. This well-established technic has an important impact on insurance practice. The present paper is devoted to the problem of finding simple analytical lower and upper bounds for stop-loss premiums and ruin probabilities of classical compound Poisson risks in case only the mean, variance and range of the claim size distribution are known.

In this situation the distributions with minimal and maximal stop-loss premiums of the claim size depend on the deductible and cannot be used to bound the stop-loss premiums or ruin probabilities of the induced compound Poisson risks uniformly for each deductible or initial reserve. However larger classes of claim size distributions may be allowed for ordering comparisons, which lead to uniform lower and upper bounds. For example Kaas and Goovaerts (1986) have derived distributions, which are the best lower and upper bound with respect to the dangerousness ordering criterion for any distribution with fixed mean, variance and range. In the present paper we derive similar distributions, which are best with respect to the stop-loss ordering criterion. They lead to uniformly better and even simpler analytical bounds than the previous ones.

In Section 2 the stop-loss ordered extremal distributions are introduced and compared with the dangerous extremal distributions. A main result states that the stop-loss order maximum precedes in dangerousness the dangerous order maximum, and that the dangerous order minimum precedes in dangerousness the stop-loss order minimum. In Section 3 improved analytical bounds for stop-loss premiums 
and ruin probabilities of compound Poisson risks are constructed through discretization of the stop-loss ordered maximal distribution applying the technic of mass dispersion. Then we comment on two special situations. In Section 4.1 the upper bounds in case of small deductibles and initial reserves are shown to coincide with the best upper bounds derived by Kaas (1991). A discussion of the obtained bounds provided the relative variance of the claim size is unknown, but its maximal value is known, is given in Section 4.2. One recovers the safest diatomic risk with fixed mean and known range from Bühlmann et al. (1977), which can be regarded as a positive answer to the following modified Schmitter problem. Given that the individual claims have given mean and maximal variance, which claims distribution maximizes the ruin probability for a given initial reserve? In case the deductible equals the mean of the compound Poisson risk, the obtained stop-loss upper bound is shown to be closely related to earlier investigations by Benktander (1977). Finally, in Section 5 the substantial improvement in the new stop-loss bounds is demonstrated numerically.

\section{STOP-LOSS ORDERED EXTREMAL VERSUS DANGEROUS EXTREMAL DISTRIBUTIONS}

Consider a risk $X$, representing claim sizes, from the set $D=D\left(I_{x} ; \mu, \sigma\right)$ of all random variables with fixed mean $\mu$, variance $\sigma^{2}$, and support contained in the interval $I_{x}=[0, b]$. By relative variance we mean the square of the coefficient of variation. The following notations are used for relative variances and a ratio thereof:

$v=(\sigma / \mu)^{2} \quad$ : relative variance of $X$

$v_{o}=(b-\mu) / \mu:$ maximal relative variance of risks with known mean $\mu$ and range $[0, b]$

$v_{r}=v / v_{o} \quad:$ ratio of relative variances

To simplify the presentation and calculations, let us work in the standardized risk scale defined by the transformation $Z=(X-\mu) / \mu$, which is interpreted as relative signed mean deviation. Then the support of $Z$ is $I_{z}=\left[-1, v_{o}\right]$.

Extremal random variables $X^{u}, X^{\ell}$, having distributions $F^{u}(x), F^{\ell}(x)$, with respect to the usual stochastic dominance partial order relation $\leq_{\mathrm{st}}$ have been given in Goovaerts and Kaas (1986):

$$
\begin{gathered}
X^{u} \leq_{s t} X \leq_{s t} X^{\ell}, \text { for all } X \in D, \\
\Leftrightarrow F^{\ell}(x) \leq F(x) \leq F^{u}(x), \text { for all } x \in I_{x} .
\end{gathered}
$$

In the transformed risk scale $Z^{\ell}=\left(X^{\ell}-\mu\right) / \mu, Z^{u}=\left(X^{u}-\mu\right) / \mu$, have distributions $F^{\ell}(z), F^{u}(z)$ as described in Table 1.

Extremal distributions $X^{-}, X^{+}$with respect to the dangerousness order relation $\leq_{D}$ are constructed in Kaas and Goovaerts (1986), and have the property $X^{-} \leq_{D} X \leq_{D} X^{+}$, for all $X \in D$. Their standardized distributions are displayed in Table 2 . 
TABLE 1

EXTREMAL DISTRIBUTIONS WITH RESPECT TO STOCHASTIC DOMINANCE

\begin{tabular}{lll}
\hline \hline & $F^{\ell}(z)$ & $F^{u}(z)$ \\
\hline$-1 \leq z \leq-v_{r}$ & 0 & $v /\left(v+z^{2}\right)$ \\
$-v_{r} \leq z \leq v$ & {$\left[v_{o} /\left(1+v_{o}\right)\right] \cdot\left(v_{r}+z\right) /(1+z)$} & {$\left[v_{o} /\left(1+v_{o}\right)\right] \cdot\left(v_{o}+1-v_{r}-z\right) /\left(v_{o}-z\right)$} \\
$v \leq z<v_{o}$ & $z^{2} /\left(v+z^{2}\right)$ & 1 \\
$z=v_{o}$ & 1 & 1 \\
\hline
\end{tabular}

Stop-loss ordered extremal distributions $X_{*}, X^{*}$, with the property $X_{*} \leq_{s l} X \leq_{s l} X^{*}$ can be constructed following an idea expressed in Hürlimann (1993/95). If $\pi_{*}(x), \pi^{*}(x), 0 \leq x \leq b$, are the minimal and maximal stop-loss premiums when $X$ varies over $D$, then these extremal distributions are defined using the one-to-one correspondence between a distribution and its stop-loss transform, namely as

$$
\begin{gathered}
F_{*}(x)=1+(d / d x) \pi_{*}(x), \text { and } \\
F^{*}(x)=1+(d / d x) \pi^{*}(x), 0 \leq x \leq b .
\end{gathered}
$$

The extreme value functions $\pi_{*}(x), \pi^{*}(x)$ have been obtained in DeVylder and Goovaerts (1982) (see also Goovaerts et al. (1984), p.316, Kaas et al. (1994), chap. X.2). After a straightforward calculation, one gets the standardized distributions of Table 3.

It may also be useful to know the expected values and variances of the dangerous and stop-loss ordered extremal distributions.

TABLE 2

DANGEROUS EXTREMAL DISTRIBUTIONS

\begin{tabular}{ll}
\hline$z$ & $F^{-}(z)$ \\
\hline$-1 \leq z \leq-v_{r}$ & 0 \\
$-v_{r} \leq z<0$ & $F^{\ell}(z)=\left[v_{o} /\left(1+v_{o}\right)\right] \cdot\left(v_{r}+z\right) /(1+z)$ \\
$0<z \leq v$ & $F^{u}(z)=\left[v_{o} /\left(1+v_{o}\right)\right] \cdot\left(v_{o}+1-v_{r}-z\right) /\left(v_{o}-z\right)$ \\
$v \leq z \leq v_{o}$ & $F^{u}(z)=1$ \\
\hline$z$ & $F^{+}(z)$ \\
\hline$-1 \leq z \leq z\left(\alpha^{+}\right)$ & $F^{u}(z)=v /\left(v+z^{2}\right)$ \\
$z\left(\alpha^{+}\right) \leq z \leq z\left(\beta^{+}\right)$ & $F^{u}\left(z\left(\alpha^{+}\right)\right)=v /\left(v+z\left(\alpha^{+}\right)^{2}\right)$ \\
$z\left(\beta^{+}\right) \leq z<v_{o}$ & $F^{\ell}(z)=z^{2} /\left(v+z^{2}\right)$ \\
$z=v_{o}$ & $F^{\ell}(z)=1$ \\
\hline$z\left(\alpha^{+}\right)=\left(\alpha^{+}-\mu\right) / \mu=\left(v-v_{r}-\left[v_{r}(1+v)\left(v_{r}+v_{o}\right)\right]_{1}^{\frac{1}{2}}\right) /\left(1+v_{r}\right)$ \\
$z\left(\beta^{+}\right)=\left(\beta^{+}-\mu\right) / \mu=\left(v-v_{r}+\left[v_{r}(1+v)\left(v_{r}+v_{o}\right)\right]^{\frac{1}{2}}\right) /\left(1+v_{r}\right)$ \\
\hline
\end{tabular}


TABLE 3

STOP-LOSS ORDERED EXTREMAL DISTRIBUTIONS

\begin{tabular}{ll}
\hline$z$ & $F_{*}(z)$ \\
\hline$-1 \leq z<-v_{r}$ & 0 \\
$-v_{r} \leq z<v$ & $v_{o} /\left(1+v_{o}\right)$ \\
$v \leq z \leq v_{o}$ & 1 \\
\hline$z$ & $F^{*}(z)$ \\
\hline$-1 \leq z \leq z\left(\alpha^{*}\right)$ & $v /(1+v)$ \\
$z\left(\alpha^{*}\right) \leq z \leq z\left(\beta^{*}\right)$ & $\frac{1}{2}\left(1+z /\left(v+z^{2}\right)^{\frac{1}{2}}\right)$ \\
$z\left(\beta^{*}\right) \leq z<v_{o}$ & $v_{o} /\left(v_{r}+v_{o}\right)$ \\
$z=v_{o}$ & 1 \\
\hline$z\left(\alpha^{*}\right)=\left(\alpha^{*}-\mu\right) / \mu=\frac{1}{2}(v-1)$ \\
$z\left(\beta^{*}\right)=\left(\beta^{*}-\mu\right) / \mu=\frac{1}{2}\left(v_{o}-v_{r}\right)$ \\
\hline
\end{tabular}

Lemma 2.1. The following expressions hold:

$$
\begin{gathered}
E\left[Z^{-}\right]=E\left[Z_{*}\right]=E\left[Z^{*}\right]=E\left[Z^{+}\right]=0 \\
\operatorname{Var}\left[Z^{-}\right]=v \cdot\left(2-v_{r}\right)+2 v_{o} \cdot\left(1-v_{r}\right) \cdot \ln \left\{1-v_{r}\right\} \\
\operatorname{Var}\left[Z_{*}\right]=v \cdot v_{r} \\
\operatorname{Var}\left[Z^{*}\right]=v \cdot\left(1-\frac{1}{2} \cdot \ln \left\{v_{r}\right\}\right) \\
\operatorname{Var}\left[Z^{+}\right]=v \cdot\left(1+\ln \left\{v \cdot\left(v_{r}+v_{o}\right) \cdot(1+v)\right\}-\ln \left\{v_{r} \cdot\left(v+z\left(\alpha^{+}\right)^{2}\right) \cdot\left(v+z\left(\beta^{+}\right)^{2}\right)\right\}\right) .
\end{gathered}
$$

Proof. The elementary calculations are left to the reader. The variances are best obtained replacing Stieltjes integrals by Riemann integrals (e.g. Kaas et al. (1994), Theorem I.3.1.1):

$$
E\left[Z^{2}\right]=1-F(-1)+2 \cdot \int_{-1}^{v o} z(1-F(z)) d z
$$

Our aim is to show the following main result.

Theorem 2.1. The dangerous and stop-loss ordered extremal random variables satisfy the following stochastic order relations between random variables with equal mean:

$$
X^{-} \leq_{D} X_{*} \leq_{s l} X \leq_{s l} X^{*} \leq_{D} X^{+} \text {, for all } X \in D .
$$

Proof. These ordering relations are a consequence of the defining ordering inequalities between $X_{*}, X$ and $X^{*}$, and the auxiliary results below, whose overall content is 


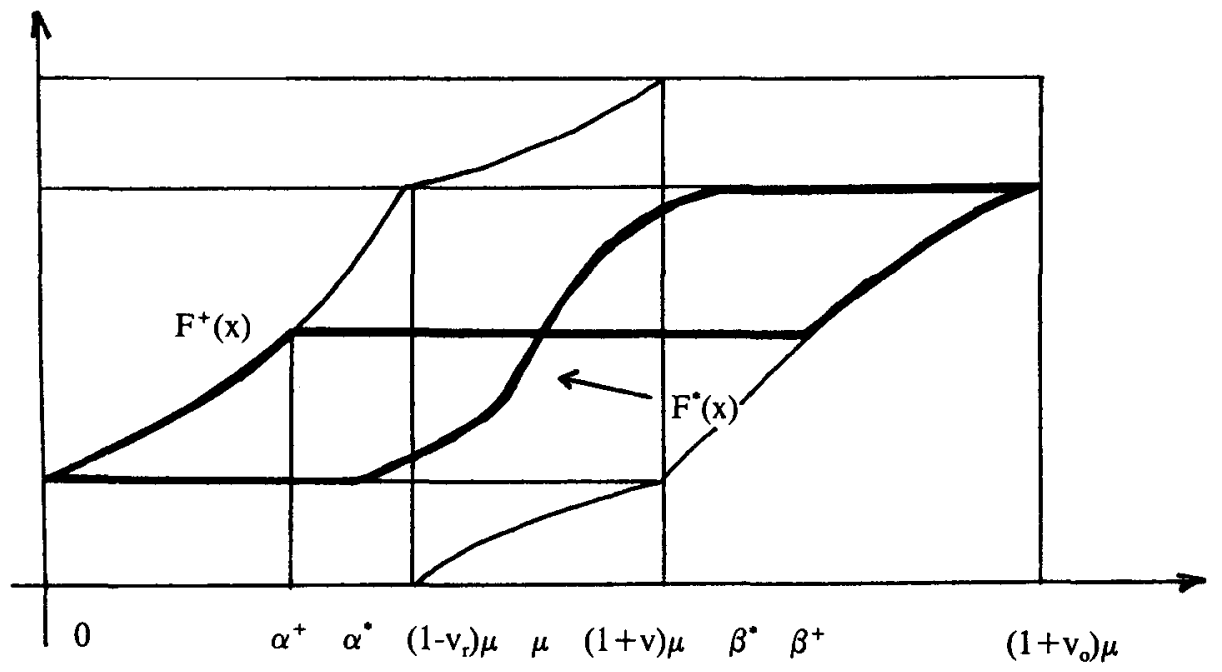

FIGURE 1 The ordering relation $X^{*} \leq_{D} X^{+}\left(\mu=2, \sigma^{2}=1, b=4\right)$

intuitively immediate from the visualizations in Figures 1 and 2, and for which a more rigorous proof is reported to the Appendix.

Lemma 2.2. The stop-loss ordered maximum satisfies the following properties:

$$
Z^{u} \leq_{s t} Z^{*} \leq_{s t} Z^{\ell} \text {, or equivalently }
$$

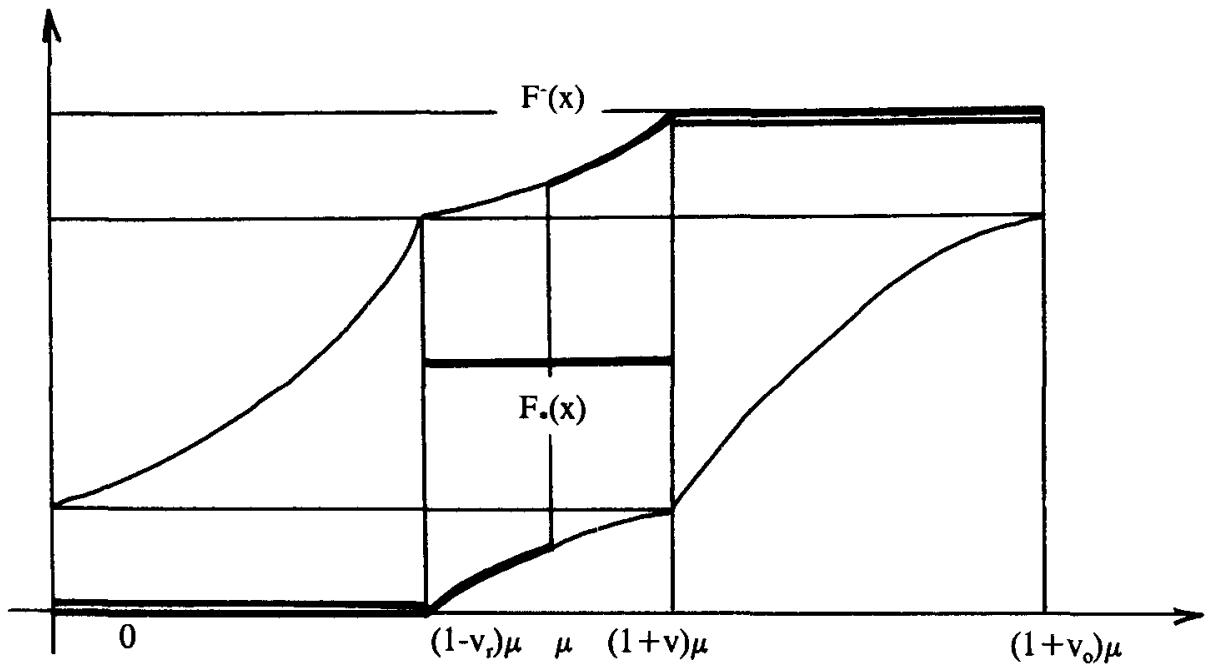

FIGURE 2 The ordering relation $X^{-} \leq_{D} X_{*}\left(\mu=2, \sigma^{2}=1, b=4\right)$ 


$$
\begin{gathered}
F^{\ell}(z) \leq F^{*}(z) \leq F^{u}(z), \text { for all } z \in I_{z}=\left[-1, v_{o}\right] . \\
F^{*}(-1)=F^{u}(-1), F^{*}\left(v_{o^{-}}\right)=F^{\ell}\left(v_{o^{-}}\right) .
\end{gathered}
$$

Lemma 2.3. The stop-loss ordered maximum is less dangerous than the dangerous maximum, that is $Z^{*} \leq_{D} Z^{+}$. More precisely one has

$$
\begin{aligned}
& F^{*}(z) \leq F^{+}(z),-1 \leq z \leq z_{o}, \\
& F^{*}(z) \geq F^{+}(z), \quad z_{o} \leq z \leq v_{o},
\end{aligned}
$$

where the crossing point is determined by

$$
z_{o}=\frac{1}{2} \sqrt{v} \cdot\left|\sqrt{v} / z\left(\alpha^{+}\right)-z\left(\alpha^{+}\right) / \sqrt{v}\right| \cdot \operatorname{sgn}\left\{1-z\left(\alpha^{+}\right)^{2} / v\right\}
$$

or in the original $x$-scale

$x_{o}=\left(1+z_{o}\right) \mu=\mu+\frac{1}{2} \sigma\left|\sigma /\left(\alpha^{+}-\mu\right)-\left(\alpha^{+}-\mu\right) / \sigma\right| \cdot \operatorname{sgn}\left\{1-\left(\alpha^{+}-\mu\right)^{2} / \sigma^{2}\right\}$.

Lemma 2.4. The stop-loss ordered minimum is more dangerous than the dangerous minimum, that is $Z^{-} \leq_{D} Z_{*}$. More precisely one has

$$
\begin{aligned}
& F^{-}(z) \leq F_{*}(z), \quad-1 \leq z \leq 0, \\
& F^{-}(z) \geq F_{*}(z), 0 \leq z \leq v_{o} .
\end{aligned}
$$

\section{BOUNDS FOR STOP-LOSS PREMIUMS AND RUIN PROBABILITIES}

An important issue in Practical Risk Theory is the construction of more or less accurate bounds on stop-loss premiums and ruin probabilities for compound random sums $S=X_{1}+\ldots+X_{N}$, where the claim number $N$ is fixed, say Poisson $(\lambda)$, and the claim sizes $X_{i}={ }_{d} X \in D$ are independent and identically distributed, $X_{i}$ independent from $N$.

\subsection{Stop-loss and ruin probability inequalities}

Since the crossing condition between dangerously ordered random variables is a sufficient condition for stop-loss order, all random variables in (2.6) are stop-loss ordered with equal means. In particular the variances of Lemma 2.1 satisfy the following inequalities

$$
\operatorname{Var}\left[X^{-}\right] \leq \operatorname{Var}\left[X_{*}\right] \leq \operatorname{Var}[X]=\sigma^{2} \leq \operatorname{Var}\left[X^{*}\right] \leq \operatorname{Var}\left[X^{+}\right]
$$

Let $S^{-}, S_{*}, S^{*}, S^{+}$the compound random sums obtained when replacing $X$ by $X^{-}$, $X_{*}, X^{*}, X^{+}$in $S$. Following Kaas (1991) (see also Kaas et al. (1994), chap. XI), the stop-loss ordering relations imply the following inequalities between stop-loss 
premiums and ultimate ruin probabilities:

$$
\begin{aligned}
& \pi\left(S^{-} ; d\right) \leq \pi\left(S_{*} ; d\right) \leq \pi(S ; d) \leq \pi\left(S^{*} ; d\right) \leq \pi\left(S^{+} ; d\right), \\
& \text { uniformly for all deductibles } d \geq 0, \text { all } X \in D, \\
& \psi\left(S^{-} ; u\right) \leq \psi\left(S_{*} ; u\right) \leq \psi(S ; u) \leq \psi\left(S^{*} ; u\right) \leq \psi\left(S^{+} ; u\right), \\
& \text { uniformly for all initial reserves } u \geq 0, \text { all } X \in D,
\end{aligned}
$$

where one uses the notations $\pi(S ; d)=E\left[(S-d)^{+}\right], \psi(S ; u)=1-\operatorname{Pr}(L \leq u)$, $L=\max \{S(t)-c t: t \geq 0\}$ the maximal aggregate loss associated to the aggregate claims up to time $t: S(t)=X_{1}+\ldots+X_{N(t)}, N(t)_{t>0}$ the Poisson process with intensity $\lambda, c=\lambda \mu(1+\theta)$ the constant premium rate with security loading $\theta \geq 0$.

In particular (3.2), (3.3) imply that the previous theoretical, numerical and analytical bounds based on the dangerous extremal risks $X^{-}, X^{+}$can be improved by using instead the stop-loss extremal risks $X_{*}, X^{*}$. For practical reasons let us restrict our attention to the evaluation of analytical bounds, which improve the previous bounds by Steenackers and Goovaerts (1991).

\subsection{Discrete approximations}

To obtain analytical bounds, discrete approximations to the claim size distribution are constructed. For $F^{-}(x), F^{+}(x)$ one finds discrete approximations $X_{d}^{-}, X_{d}^{+}$in Steenackers and Goovaerts (1991). Since $F_{*}(x)$ is already a diatomic distribution, it remains to discuss the discretization of $F^{*}(x)$.

By means of mass dispersion over the interval $\left[z\left(\alpha^{*}\right), z\left(\beta^{*}\right)\right]$, let us construct the following 4-atomic random variable $Z_{d}^{*}=\left\{-1, z\left(\alpha^{*}\right), z\left(\beta^{*}\right), v_{o}\right\}$, which is necessarily more dangerous than $Z^{*}$, and thus also stop-loss larger (see e.g. Gerber (1979), chap.7, Example 3.2). The probabilities of $Z_{d}^{*}$ are defined by the following conditions:

$$
\begin{gathered}
p(-1)=F^{*}(-1)=v /(1+v) \\
p\left(\alpha^{*}\right)+p\left(\beta^{*}\right)=F^{*}\left(z\left(\beta^{*}\right)\right)-F^{*}\left(z\left(\alpha^{*}\right)\right) \\
z\left(\alpha^{*}\right) \cdot p\left(\alpha^{*}\right)+z\left(\beta^{*}\right) \cdot p\left(\beta^{*}\right)=\int_{z\left(\alpha^{*}\right)}^{z\left(\beta^{*}\right)} z d F^{*}(z) \\
p\left(v_{o}\right)=1-F^{*}\left(z\left(\beta^{*}\right)\right)=v_{r} /\left(v_{r}+v_{o}\right)
\end{gathered}
$$

The condition (3.5) preserves the probability mass over $\left[z\left(\alpha^{*}\right), z\left(\beta^{*}\right)\right]$ while (3.6) preserves the mean. The right-hand side in (3.6) equals

$$
\left[-\left(1-F^{*}(Z)\right)\right]_{z\left(\alpha^{*}\right)}^{z\left(\beta^{*}\right)}+\int_{z\left(\alpha^{*}\right)}^{z\left(\beta^{*}\right)}\left(1-F^{*}(z)\right) d z
$$


where the first term is

$$
\frac{1}{2}(v-1) /(v+1)-\frac{1}{2}\left(v-v_{r}\right) /\left(v_{o}+v_{r}\right)
$$

and the second one is

$$
\frac{1}{2}\left[z-\left(v+z^{2}\right)^{\frac{1}{2}}\right]_{z\left(\alpha^{*}\right)}^{z\left(\beta^{*}\right)}=\frac{1}{2}\left(1-v_{r}\right)
$$

where use has been made of the relations

$$
v+z\left(\alpha^{*}\right)^{2}=\frac{1}{4}(v+1)^{2}, v+z\left(\beta^{*}\right)^{2}=\frac{1}{4}\left(v_{o}+v_{r}\right)^{2} .
$$

It remains to solve the linear system of equations

$$
\begin{aligned}
& p\left(\alpha^{*}\right)+p\left(\beta^{*}\right)=A, z\left(\alpha^{*}\right) \cdot p\left(\alpha^{*}\right)+z\left(\beta^{*}\right) \cdot p\left(\beta^{*}\right)=B, \text { with } \\
& A=v_{o} /\left(v_{o}+v_{r}\right)-v /(v+1), B=v /(1+v)-v /\left(v_{r}+v_{o}\right) .
\end{aligned}
$$

A calculation shows that

$$
\begin{aligned}
& p\left(\alpha^{*}\right)=\left[A z\left(\beta^{*}\right)-B\right] /\left[z\left(\beta^{*}\right)-z\left(\alpha^{*}\right)\right]=\left(v_{o}-v\right) /\left[\left(1+v_{o}\right) \cdot(1+v)\right] \\
& p\left(\beta^{*}\right)=\left[B-A z\left(\alpha^{*}\right)\right] /\left[z\left(\beta^{*}\right)-z\left(\alpha^{*}\right)\right]=\left(v_{o}-v\right) /\left[\left(1+v_{o}\right) \cdot\left(v_{r}+v_{o}\right)\right] .
\end{aligned}
$$

Since $v \leq v_{o}$ the probabilities are always non-negative, as should be.

\subsection{The analytical bounds}

A compound Poisson $(\lambda)$ risk $S$ with discrete claim size support $X=\left\{0, x_{1}, x_{2}, x_{3}\right\}$, and probabilities $p_{0}, p_{1}, p_{2}, p_{3}$ can be expressed as (e.g. Gerber (1979), chap.1, Section 7, or Bowers et al. (1986), Theorem 11.2):

$$
S=x_{1} N_{1}+x_{2} N_{2}+x_{3} N_{3}
$$

where the numbers of occurrences $N_{i}$ of claim size $x_{i}$ are independent Poisson with parameter $\lambda p_{i}, i=1,2,3$. From this representation one gets after some well-known calculations the needed analytical formulas:

$$
\begin{aligned}
\pi(S ; d)= & E\left[(S-d)^{+}\right]=\lambda \mu-d \\
& +\exp \left\{-\lambda\left(1-p_{0}\right)\right\} \sum_{n i=0}^{\infty}\left[\left(\lambda p_{1}\right)^{n 1} /\right. \\
& \left.n_{1} !\left(\lambda p_{2}\right)^{n 2} / n_{2} !\left(\lambda p_{3}\right)^{n 3} / n_{3} !\right] \cdot\left(d-\sum_{i=1}^{3} n_{i} x_{i}\right)^{+}
\end{aligned}
$$




$$
\begin{aligned}
\psi(S ; u)= & 1-\operatorname{Pr}(\mathrm{L} \leq u)= \\
& 1-\theta /(1+\theta) \sum_{n i=0}^{\infty}\left[p_{1}^{n 1} / n_{1} ! p_{2}^{n 2} / n_{2} ! p_{3}^{n 3} / n_{3} !\right] \cdot \exp \left\{z\left(1-p_{0}\right)\right\} \cdot(-z)^{n 1+n 2+n 3}, \\
& \text { with } z=\left(u-\sum_{i=1}^{3} n_{i} x_{i}\right)^{+} / \mu(1+\theta)
\end{aligned}
$$

It is important to observe that these infinite series representations are always finite sums because summation occurs only for $\sum_{i=1}^{3} n_{i} x_{i}<d, u$.

Table 4 provides a unified overview of the discrete approximations used to get analytical bounds for compound Poisson risks. A numerical illustration, which demonstrates the obtained substantial improvement is found in Section 5.

\section{SPECial CASES}

It is interesting and useful to relate our results to various other considerations made so far in the actuarial literature. We illustrate with two examples.

\subsection{The upper bounds for small deductibles and initial reserves}

Suppose the deductible of a stop-loss contract is small such that $d \leq \alpha^{*}=\frac{1}{2}(1+v) \mu$. Then the infinite series representation (3.17) shrinks to the only term $n_{1}=n_{2}=n_{3}=0$, and therefore one has

$$
\pi\left(S_{d}^{*} ; d\right)=\lambda \mu-d+\exp \left\{-\lambda\left(1-p_{0}\right)\right\} \cdot d .
$$

In terms of the mean $\mu_{s}=\lambda \mu$ and the relative variance $v_{s}=(1+v) \lambda$ of a compound Poisson $(\lambda)$ risk $S$ with claim size $X \in D$, this can be rewritten as

$$
\left.\pi\left(S_{d}^{*} ; d\right)=\lambda \mu-d+\exp \left\{-1 / v_{s}\right)\right\} \cdot d, d \leq \frac{1}{2}(1+v) \mu .
$$

From Kaas (1991), p.141, one knows that the maximizing claim size distribution over $D$ is the diatomic risk with support $\left\{x_{1}, x_{2}\right\}=\{0,(1+v) \mu\}$ and probabilities $p_{1}=v /(1+v), \quad p_{2}=1 /(1+v)$. The corresponding (compound) Poisson risk $S=(1+v) \mu N, \quad N$ Poisson $(\lambda)$, has the same stop-loss premiums $\pi(S ; d)=\pi\left(S_{d}^{*} ; d\right)$ provided $d \leq \frac{1}{2}(1+v) \mu$. A similar result holds for the ultimate ruin probabilities. Therefore our analytical upper bounds obtained from the stop-loss ordered maximal distribution coincides in the special case of small deductibles and initial reserves with the optimal (=best upper) bounds.

\subsection{The bounds by unknown relative variance}

Suppose only the mean $\mu$ and the maximal relative variance $v_{o}$ are known, but the true relative variance $v$ is unknown. Equivalently $\mu$ and the upper end point $b=\left(1+v_{o}\right) \mu$ of the interval $I_{x}$ are known. The true $v$ satisfies the inequality $0 \leq v \leq v_{o}$. Choosing $v=v_{o}$ in the formulas determining $X_{d}^{*}$, one gets $\alpha^{*}=\beta^{*}=\frac{1}{2}\left(1+v_{o}\right) \mu, p\left(\alpha^{*}\right)=p\left(\beta^{*}\right)=0$, and thus $X_{d}^{*}$ is a diatomic risk with 
TABLE 4

DisCRETE CLAIM SIZE APPROXIMATIONS

\begin{tabular}{lllll}
\hline \hline claim size & $X_{d}^{-}$ & $X_{*}$ & $X_{d}^{*}$ & $X_{d}^{+}$ \\
\hline atoms & 3 & 2 & 4 & 4 \\
\hline$x_{1}$ & $-\mu \ln \left\{1-v_{r}\right\}\left(1-v_{r}\right) / v_{r}$ & $\mu\left(1-v_{r}\right)$ & $\alpha^{*}$ & $\alpha^{+}$ \\
$x_{2}$ & $\mu$ & $\mu(1+v)$ & $\beta^{*}$ & $\beta^{+}$ \\
$x_{3}$ & $\mu\left(1+v_{o}\right)+$ & $d+1$, or & $\mu\left(1+v_{o}\right)$ & $\mu\left(1+v_{o}\right)$ \\
& $\mu v_{o} \ln \left\{1-v_{r}\right\}\left(1-v_{r}\right) / v_{r}$ & $u+1$ & & \\
\hline$p_{0}$ & 0 & 0 & $v /(1+v)$ & $\sqrt{v}\left(\mu / x_{1}\right) \operatorname{Arctan}\left\{\sqrt{v}\left(x_{1} / \mu\right) /\right.$ \\
& & & & $\left.\left(v+1-\left(x_{1} / \mu\right)\right)\right\}$ \\
$p_{1}$ & $v_{r} v_{o} /\left(1+v_{o}\right)$ & $v_{o} /\left(1+v_{o}\right) p\left(\alpha^{*}\right)$ & $v /\left(v+\left(x_{1} / \mu-1\right)^{2}\right)-p_{0}$ \\
$p_{2}$ & $1-v_{r}$ & $1 /\left(1+v_{o}\right)$ & $p\left(\beta^{*}\right)$ & $1-p_{0}-p_{1}-p_{3}$ \\
$p_{3}$ & $v_{r} /\left(1+v_{o}\right)$ & 1 & $v_{r} /\left(v_{r}+v_{o}\right)$ & $\sqrt{v} /\left(v_{o}+1-x_{2} / \mu\right)$ \\
& & & & Arctan $\left\{\sqrt{v} \cdot\left(v_{o}+1-x_{2} / \mu\right)\right.$ \\
& & & & $\left./\left(v+v_{o}\left(x_{2} / \mu-1\right)\right)\right\}$ \\
\hline
\end{tabular}

$\alpha^{*}=\frac{1}{2} \mu(1+v), \beta^{*}=\mu\left(1+\frac{1}{2}\left(v_{o}-v_{r}\right)\right)$

$p\left(\alpha^{*}\right)=\left(v_{o}-v\right) /\left[\left(1+v_{o}\right)(1+v)\right], p\left(\beta^{*}\right)=\left(v_{o}-v\right) /\left[\left(1+v_{o}\right)\left(v_{r}+v_{o}\right)\right]$

$\alpha^{+}=\mu\left\{1+\left[v-v_{r}-\left(v_{r}(1+v)\left(v_{r}+v_{o}\right)\right)_{1}^{\frac{1}{2}}\right] /\left(1+v_{r}\right)\right\}$

$\beta^{+}=\mu\left\{1+\left[v-v_{r}+\left(v_{r}(1+v)\left(v_{r}+v_{o}\right)\right)^{\frac{1}{2}}\right] /\left(1+v_{r}\right)\right\}$

support $\{0, b\}$ and probabilities $p(0)=v_{o} /\left(1+v_{o}\right), p(b)=1 /\left(1+v_{o}\right)$. Similarly $X_{*}$ goes over to the same diatomic risk. In both cases one recovers the safest risk with fixed mean $\mu$ and range $\left[0, b=\left(1+v_{o}\right) \mu\right]$ (e.g. Bühlmann et al. (1977), Kaas et al. (1994), Example III.1.2). As a consequence, if $\mu$ and $v_{o}$ are known, but $v$ is unknown, compound Poisson $(\lambda)$ stop-loss premiums should be calculated according to the simple formula

$$
\begin{aligned}
\pi\left(S_{d}^{*} ; d\right) & =\mu_{s}-d+\exp \left\{-1 / v_{o, s}\right\} \sum_{n=0}^{n o(d)}\left(d-n v_{o, s} \mu_{s}\right) /\left[\left(v_{o, s}\right)^{2 n} \cdot n !\right], \text { with } \\
n o(d) & =\left[d / v_{o, s} \mu_{s}\right]([x]: \text { greatest integer less than } x),
\end{aligned}
$$

where $v_{o, s}=\left(1+v_{o}\right) / \mu$ denotes the maximal relative variance of a compound Poisson $(\lambda)$ risk with mean claim size $\mu$ and maximal relative variance $v_{o}$ of the claim size. Similarly the ultimate ruin probability should be calculated as

$$
\begin{aligned}
\psi\left(S_{d}^{*} ; u\right) & =1-\theta /(1+\theta) \sum_{m=0}^{m o(u)} \exp \left\{z / v_{o, s}\right\}\left(-z / v_{o, s}\right)^{m} / m !, \text { with } \\
z & =\left(u-m v_{o, s} \mu_{s}\right) /(1+\theta) \mu_{s}, m o(u)=\left[u / v_{o, s} \mu_{s}\right] .
\end{aligned}
$$

In particular the latter formula can be viewed as a positive answer to the following modified Schmitter problem (discussion papers on this topic are Brockett et al. (1991) and Kaas (1991)). Given that the individual claims have mean $\mu$ and 
maximal variance $v_{o} \mu^{2}$, which claims distribution maximizes the ruin probability for a given initial reserve $u$ ?

On the other side it is interesting to look at the special deductible $d=\mu_{s}$, the mean of a compound Poisson $(\lambda)$ risk. In this situation the safest stop-loss upper bound (4.3) can be rewritten as

$$
\begin{aligned}
\pi\left(S_{d}^{*} ; \mu_{s}\right) & =\mu_{s} \cdot \exp \left(-\lambda_{o}+\left[\lambda_{o}\right] \ln \left\{\lambda_{o}\right\}\right) /\left[\lambda_{o}\right] ! \\
& \approx \mu_{s} \cdot \exp \left(-\lambda_{o}+\lambda_{o} \ln \left\{\lambda_{o}\right\}\right) / \Gamma\left(\lambda_{o}+1\right), \lambda_{o}=1 / v_{o, s} .
\end{aligned}
$$

This is the special stop-loss premium of a compound Poisson $\left(\lambda_{o}\right)$ risk with individual claims of equal size $\mu_{s} v_{o, s}$. It is approximately equal to the special stop-loss premium of a $\operatorname{Gamma}\left(\lambda_{o}, \lambda_{o} / \mu_{s}\right)$ distributed risk with mean $\mu_{s}$ and variance $v_{o, s} \mu_{s}^{2}$. Applying Stirling's approximation formula for the Gamma function

$$
\Gamma\left(\lambda_{o}+1\right) \approx \exp \left(-\lambda_{o}+\lambda_{o} \ln \left\{\lambda_{o}\right\}\right)\left(2 \pi \lambda_{o}\right)^{\frac{1}{2}} \cdot\left(1+1 / 12 \lambda_{o}+\ldots\right),
$$

one gets approximately

$$
\pi\left(S_{d}^{*} ; \mu_{s}\right) \approx \mu_{s}\left(2 \pi \lambda_{o}\right)^{-\frac{1}{2}} /\left(1+1 / 12 \lambda_{o}+\ldots\right)<\mu_{s}\left(v_{o, s} / 2 \pi\right)^{\frac{1}{2}} .
$$

The upper bound on the right-hand side is the special stop-loss premium of a Normal $\left(\mu_{s}, v_{o, s} \mu_{s}^{2}\right)$ distributed risk, to which $\pi\left(S_{d}^{*} ; \mu_{s}\right)$ converges when the relative variance $v_{o, s}$ goes to zero. The latter property is known to be true asymptotically (that is in large portfolios) for arbitrary compound Poisson risks (e.g. Daykin et al. (1994), p.64). Note that the above investigation corresponds to the findings of Benktander (1977), where as an important additional complement, the parameter

$$
1 / \lambda_{o}=v_{o, s}=\left(1+v_{o}\right) / \lambda
$$

should be equal to the maximal relative variance of the considered compound Poisson risk, given that the true relative variance is unknown.

\section{NUMERICAL ILLUSTRATION}

Though it would be possible to compute close numerical lower and upper bounds to the considered risk quantities for all of the compound Poisson risks with claim sizes $X^{-}, X_{*}, X^{*}, X^{+}$, we restrict our numerical investigation to a comparison of the corresponding analytical bounds as summarized in Table 4.

The same examples as in Steenackers and Goovaerts (1991) have been calculated. The claim size distribution is assumed to be uniform $(1,3)$ with range $[0,3]$, hence $\mu=2, v=1 / 12, v_{o}=1 / 2, v_{r}=1 / 6$, and the Poisson parameter $\lambda$ is 1 , or 10 . The safety loading is $\theta=0.2$. The results are found in Tables $5,6,7$. The improvement is very substantial for the new stop-loss bounds, but less spectacular for the ruin probabilities. Concerning the previous bounds, small numerical inaccuracies for the deductible $d=60$ in Table 6 , as well as in Table 7 have been located, probably due to rounding errors. 
TABLE 5

BOUNDS FOR STOP-LOSS PREMIUMS WITH CLAIM-RANGE $[0,3]$, MEAN 2, VARIANCE $1 / 3$ AND CLAIM NUMBER POISSON (1)

\begin{tabular}{llllll}
\hline \hline $\mathrm{d}$ & exact value & bound $X_{d}^{-}$ & bound $X_{*}$ & bound $X_{d}^{*}$ & bound $X_{d}^{+}$ \\
\hline 2 & $8.277 \cdot 10^{-1}$ & $89.3 \%$ & $93.8 \%$ & $107.3 \%$ & $113.9 \%$ \\
4 & $2.689 \cdot 10^{-1}$ & $78.4 \%$ & $87.2 \%$ & $115.7 \%$ & $127.2 \%$ \\
6 & $7.184 \cdot 10^{-2}$ & $67.2 \%$ & $77.6 \%$ & $122.7 \%$ & $138.1 \%$ \\
8 & $1.627 \cdot 10^{-2}$ & $56.6 \%$ & $72.1 \%$ & $141.9 \%$ & $188.6 \%$ \\
10 & $3.253 \cdot 10^{-3}$ & $46.0 \%$ & $63.0 \%$ & $164.3 \%$ & $237.8 \%$ \\
12 & $5.815 \cdot 10^{-4}$ & $36.5 \%$ & $51.9 \%$ & $190.9 \%$ & $279.2 \%$ \\
14 & $9.346 \cdot 10^{-5}$ & $28.5 \%$ & $44.6 \%$ & $220.2 \%$ & $388.5 \%$ \\
16 & $1.366 \cdot 10^{-5}$ & $21.9 \%$ & $36.5 \%$ & $260.1 \%$ & $533.2 \%$ \\
18 & $1.840 \cdot 10^{-6}$ & $16.5 \%$ & $28.4 \%$ & $322.0 \%$ & $670.0 \%$ \\
20 & $2.302 \cdot 10^{-7}$ & $12.2 \%$ & $22.9 \%$ & $379.2 \%$ & $934.5 \%$ \\
\hline
\end{tabular}

\section{APPENDIX}

Proof of Lemma 2.2. (2.9) is immediate. Let us show (2.8) in two steps.

Step 1: $F^{\ell}(z) \leq F^{*}(z)$

(a) $-1 \leq z \leq v$ :

$$
F^{\ell}(z) \leq F^{\ell}(v)=v /(1+v)=F^{*}(-1) \leq F^{*}(z)
$$

(b) $z \geq v$ :

(b1) $v_{o} \geq(1+\sqrt{2}) \sqrt{v}\left(\Rightarrow v \leq \frac{1}{2}\left(v_{o}-v_{r}\right)\right)$

TABLE 6

BOUNDS FOR STOP-LOSS PREMIUMS WITH CLAIM-RANGE [0,3], MEAN 2, VARIANCE $1 / 3$ AND Claim NUMBer PoISSON (10)

\begin{tabular}{llllll}
\hline $\mathrm{d}$ & exact value & bound $X_{d}^{-}$ & bound $X_{*}$ & bound $X_{d}^{*}$ & bound $X_{d}^{+}$ \\
\hline 15 & 5.757 & $99.0 \%$ & $99.1 \%$ & $101.7 \%$ & $103.3 \%$ \\
20 & 2.626 & $95.6 \%$ & $96.7 \%$ & $105.4 \%$ & $110.1 \%$ \\
25 & $9.321 \cdot 10^{-1}$ & $91.6 \%$ & $92.3 \%$ & $112.5 \%$ & $123.3 \%$ \\
30 & $2.563 \cdot 10^{-1}$ & $81.8 \%$ & $85.7 \%$ & $123.6 \%$ & $145.3 \%$ \\
35 & $5.507 \cdot 10^{-2}$ & $74.7 \%$ & $77.4 \%$ & $139.9 \%$ & $179.7 \%$ \\
40 & $9.383 \cdot 10^{-3}$ & $61.0 \%$ & $68.1 \%$ & $162.6 \%$ & $232.3 \%$ \\
45 & $1.289 \cdot 10^{-3}$ & $53.1 \%$ & $58.2 \%$ & $193.7 \%$ & $313.4 \%$ \\
50 & $1.449 \cdot 10^{-4}$ & $40.0 \%$ & $48.4 \%$ & $236.1 \%$ & $439.0 \%$ \\
55 & $1.355 \cdot 10^{-5}$ & $33.3 \%$ & $39.2 \%$ & $293.6 \%$ & $634.2 \%$ \\
60 & $1.067 \cdot 10^{-6}$ & $23.3 \%$ & $31.2 \%$ & $371.8 \%$ & $945.9 \%$ \\
\hline
\end{tabular}


TABLE 7

BOUNDS FOR RUIN PROBABILITIES WITH CLAIM-RANGE [0,3], MEAN 2, VARIANCE $1 / 3$ AND SAFETY MARGIN $20 \%$

\begin{tabular}{lllll}
\hline \hline$u$ & bound $X_{d}^{-}$ & bound $X_{*}$ & bound $X_{d}^{*}$ & bound $X_{d}^{+}$ \\
\hline 1 & 0.747184 & 0.747184 & 0.755158 & 0.764982 \\
2 & 0.617238 & 0.625370 & 0.663538 & 0.682110 \\
3 & 0.523757 & 0.526666 & 0.566954 & 0.587361 \\
4 & 0.437362 & 0.441446 & 0.492510 & 0.517595 \\
5 & 0.366885 & 0.371088 & 0.425256 & 0.452560 \\
6 & 0.307327 & 0.311606 & 0.367586 & 0.395577 \\
7 & 0.257467 & 0.261752 & 0.317711 & 0.346172 \\
8 & 0.215718 & 0.219854 & 0.274574 & 0.302710 \\
9 & 0.180725 & 0.184666 & 0.237313 & 0.264791 \\
10 & 0.151413 & 0.155110 & 0.205100 & 0.231598 \\
20 & 0.025798 & 0.027111 & 0.047693 & 0.060689 \\
30 & 0.004396 & 0.004739 & 0.011090 & 0.015903 \\
40 & 0.000749 & 0.000828 & 0.002579 & 0.004167 \\
50 & 0.000130 & 0.000149 & 0.000600 & 0.001092 \\
\hline
\end{tabular}

If $\frac{1}{2}(v-1) \leq v \leq z \leq \frac{1}{2}\left(v_{o}-v_{r}\right)$ one checks that

$$
F^{\ell}(z)=z^{2} /\left(v+z^{2}\right) \leq F^{*}(z)=\frac{1}{2}\left(1+z /\left(v+z^{2}\right)^{\frac{1}{2}}\right)
$$

If $v \leq \frac{1}{2}\left(v_{o}-v_{r}\right) \leq z<v_{o}$ one has

$$
F^{\ell}(z) \leq F^{\ell}\left(v_{o}-\right)=v_{o} /\left(v_{r}+v_{o}\right)=F^{*}(z)
$$

(b2) $v_{o} \leq(1+\sqrt{2}) \sqrt{v}\left(\Rightarrow v \geq \frac{1}{2}\left(v_{o}-v_{r}\right)\right)$

One concludes as in the second if-part of (b1)

Step 2: $F^{*}(z) \leq F^{u}(z)$

(a) $-1 \leq z \leq-v_{r}$ :

(al) $1 / v_{o} \leq \frac{1}{2}(1-v) / v\left(\Rightarrow \frac{1}{2}(v-1) \leq-v_{r}\right)$ If $-1 \leq z \leq \frac{1}{2}(v-1)$ then one has

$$
F^{*}(z)=v /(1+v)=F^{u}(-1) \leq F^{u}(z)
$$

If $\frac{1}{2}(v-1) \leq z \leq-v_{r} \leq \frac{1}{2}\left(v_{o}-v_{r}\right)$ one checks that

$$
F^{*}(z)=\frac{1}{2}\left(1+z /\left(v+z^{2}\right)^{\frac{1}{2}}\right) \leq v /\left(v+z^{2}\right)=F^{u}(z)
$$

(a2) $1 / v_{o} \geq \frac{1}{2}(1-v) / v\left(\Rightarrow \frac{1}{2}(v-1) \geq-v_{r}\right)$

One concludes as in the first if-part of (al)

(b) $-v_{r} \leq z \leq v_{o}$ :

$$
F^{*}(z) \leq F^{*}\left(v_{o}-\right)=v_{o} /\left(v_{r}+v_{o}\right)=F^{u}\left(-v_{r}\right) \leq F^{u}(z)
$$


Proof of Lemma 2.3. From Lemma 2.2 and the expression for $F^{+}(z)$ one has

$$
\begin{gathered}
F^{\ell}(z) \leq F^{*}(z) \leq F^{u}(z), \text { for all } z \in\left[-1, v_{o}\right) \\
F^{*}(-1)=F^{+}(-1), F^{*}\left(v_{o}-\right)=F^{+}\left(v_{o}-\right) .
\end{gathered}
$$

Since $F^{*}(z)$ is continuous and non-decreasing on $\left[-1, v_{o}\right)$, it follows from undergraduate calculus that $F^{*}(z)$ takes any value between $F^{+}(-1)$ and $F^{+}\left(v_{o}-\right)$. In particular there exists $z_{o}$ such that $F^{*}\left(z_{o}\right)=F^{u}\left(z\left(\alpha^{+}\right)\right)$. By construction of $F^{+}(z)$, one obtains

$$
\begin{aligned}
& F^{*}(z) \leq F^{+}(z),-1 \leq z \leq z_{o} \\
& F^{*}(z) \geq F^{+}(z), z_{o} \leq z \leq v_{o}
\end{aligned}
$$

which shows that $Z^{*} \leq_{D} Z^{+}$. The crossing point $z_{o}$ is obtained by solving its defining equation.

Proof of Lemma 2.4. This is immediately seen from the expressions for $F^{-}(z), F_{*}(z)$ given in Tables 2 and 3.

Note added in proof. After this paper has been submitted, the author has found the stop-loss ordered extremal distributions of Table 3 in Stoyan (1973).

\section{REFERENCES}

BENKTANDER, G. (1977). On the rating of a special stop-loss cover. ASTIN Bulletin 9, 33-41.

Bowers, N.L., Gerber, H.U., Hickman, J.C., Jones, D.A., Nesbitt, C.J. (1986). Actuarial Mathematics. Society of Actuaries.

Brockett, P., GoovaerTs, M.J., Taylor, G. (1991). The Schmitter problem. ASTIN Bulletin 21, 129-32.

Bühlmann, H., Gagliardi, B., Gerber, H.U., Straub, E. (1977). Some inequalities for stop-loss premiums. ASTIN Bulletin 9, 75-83.

Daykin, C.D., PentikäInen, T., Pesonen, M. (1994). Practical risk theory for actuaries. Monographs on Statistics and Applied Probability 53, Chapman and Hall.

DEVyLder, F., GoovaERTS, M.J. (1982). Upper and lower bounds un stop-loss premiums in case of known expectation and variance of the risk variable. Bulletin of the Swiss Association of Actuaries, 149-64.

GERBER, H.U. (1979). An introduction to mathematical risk theory. Huebner Foundation, Irwin.

Goovaerts, M.J., DeVylder, F., HaezendonCK, J. (1984). Insurance premiums. North-Holland.

GoovaerTs, M.J., KaAs, R. (1986). Best bounds for positive distributions with fixed moments. Insurance: Mathematics and Economics, 87-92.

HÜrLIMANN, W. (1993). Solvabilité et réassurance. Bulletin of the Swiss Association of Actuaries, 229-49.

HürlimanN, W. (1995). A stop-loss ordered extremal distribution and some of its applications. XXVI-th ASTIN Colloquium, Leuven, September 1995.

KAAS, R. (1991). The Schmitter problem and a related problem: a partial solution. ASTIN Bulletin 21, 13346.

KAAS, R., GoovaERTS, M.J. (1986). Bounds on stop-loss premiums for compound distributions. ASTIN Bulletin 16, 13-17.

KaAs, R., Heerwanden, A.E. van, Goovaerts, M.J. (1994). Ordering of actuarial risks. Caire Education Series 1, Brussels. 
Steenackers, A., Goovaerts, M.J. (1991). Bounds on stop-loss premiums and ruin probabilities. Insurance: Mathematics and Economics 10, 153-59.

Stoyan, D. (1973). Bounds for the extrema of the expected value of a convex function of independent random variables. Studia Scientiarum Mathematicarum Hungarica 8, 153-59. 